Photo credits

Front cover photographs: top left; OiStockphoto.com/Yegor Korzh UNOV and UNODC Photo Libraries 
Laboratory and Scientific Section

UNITED NATIONS OFFICE ON DRUGS AND CRIME

Vienna

\section{Glossary of Terms for Quality Assurance and Good Laboratory Practices}

A commitment to quality and continuous improvement

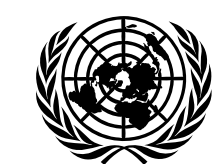

UNITED NATIONS

New York, 2009 


\section{Acknowledgements}

This manual was produced by the Laboratory and Scientific Section (LSS) of the United Nations Office on Drugs and Crime (UNODC) and its revision was coordinated by Iphigenia Naidis, staff of UNODC LSS (headed by Justice Tettey).

LSS wishes to express its appreciation and thanks to Dr. Robert Anderson, member of the Standing Panel of the UNODC's International Quality Assurance Programme, for the review and finalization of the document, as well as to the other members of the Standing Panel, Dr. Robert Bramley, Dr. David Clarke, and Dr. Pirjo Lillsunde, for their valuable contributions to this revision.*

*Contact details of named individuals can be requested from the UNODC Laboratory and Scientific Section (PO Box 500, 1400 Vienna, Austria).

ST/NAR/26/Rev.1

UNITED NATIONS PUBLICATION

Sales No. E.10.XI.7

ISBN 978-92-1-148253-9

This publication has not been formally edited. 


\section{Introduction}

\section{A. Background}

The international nature of the drug and crime problem requires the quality of the analyses and results of national laboratories as they have significant implications for the justice system, law enforcement, crime and drug prevention and health policy, as well as for the international harmonization and worldwide exchange of information and data.

Development of internationally acceptable analytical methods of detection and assay contributes greatly towards the achievement of these objectives. In addition, recognizing the importance of analytical accuracy, it is increasingly recommended that laboratories should implement quality assurance procedures, including participation in programmes which provide proficiency testing and laboratory accreditation, and that they should adopt the use of Good Laboratory Practices or adherence to ISO/IEC 17025:2005 [1].

In achieving this aim, UNODC has been publishing a series of manuals for use by national laboratories dealing with methods for the analysis of drugs in seized materials and biological specimens, and continuously seeks to provide assistance to national laboratories in quality assurance matters. This includes the provision of new manuals to help laboratories meet new developments in this field and also by updating the existing manuals. Recently, two guidance manuals have been published, on the Implementation of a Quality Management System in Drug Testing Laboratories [1] and on Validation of Analytical Methodology and Calibration of Equipment used for Testing of Illicit Drugs in Seized Materials and Biological Specimens [2].

The current publication is an update of the first edition of the Glossary of Quality Assurance Terms [3]. This second edition includes many new terms which have appeared in UNODC publications since the Glossary was first published.

\section{B. Purpose of the Glossary}

The field of quality assurance is beset by an extensive and sometimes confusing terminology, the use of which has still not been universally agreed on or accepted by 
laboratories or interested professional bodies worldwide. Several organizations, including ISO/IEC, IUPAC, Eurachem and SWDRG have standardized the use of certain terms in an attempt to clarify their application $[4,5,6]$.

This glossary contains some of the most commonly used expressions in these fields, including those which appear in UNODC publications on quality assurance, and is an attempt to help promote and harmonize national efforts by providing internationally acceptable terminology. It is intended to be an aid to analysts in the implementation of quality assurance programmes and good laboratory practices, and is provided to laboratories as an educational document, and as a means of encouraging laboratories to collaborate and participate in quality assurance matters. As a result, the Glossary includes more than one definition of a word or phrase where these might improve understanding of what they mean.

\section{Use of the Glossary}

The glossary is intended to be used in conjunction with UNODC publications on quality assurance and good laboratory practices. Attention is drawn to the importance of the availability of textbooks on quality assurance, including reference books on statistical procedures used in quality assurance. Many documents are now available in electronic format, which can be downloaded over the Internet from the websites of relevant organizations, including all UNODC publications. Furthermore, the analyst is expected to keep abreast of developments in the field by following current literature on the subject.

The Laboratory and Scientific Section of UNODC would welcome observations on the contents and usefulness of this glossary. Comments may be addressed to:

Laboratory and Scientific Section

United Nations Office on Drugs and Crime

Vienna International Centre, VIC

PO Box 500

1400 Vienna

Austria

Fax: (+43-1) 26060-5967

Email: lab@unodc.org

Website: www.unodc.org 


\section{Glossary}

Note: Words printed in italics indicate those which are glossary entries.

These definitions are intended to update the first edition of the (ST/NAR/26) [3]. They consist of terms introduced in subsequent UNODC publications which do not appear in the original Glossary. Furthermore, additional terms, definitions and explanations are taken from the SWGDRUG Glossary and the EURACHEM Guide on The Fitness for Purpose of Analytical Methods [6,7]. References to the sources of definitions can be found in these sources, if not given below.

\section{Absolute error: See Error.}

Acceptance criteria: Conditions which must be fulfilled before an operation, process or item, such as a piece of equipment, is considered to be satisfactory or to have been completed in a satisfactory way [8]. Specific examples are given below.

Acceptance criteria for software: Criteria a software product must meet to complete successfully a test phase or to achieve delivery requirements [9].

Acceptance criteria for specimens: Procedures for acceptance or rejection of specimens arriving at the analytical laboratory. Such procedures are focused on assessing the adequacy of chain of custody [8].

Accountability: Quality of subordinate workers being responsible for their own work and answerable to a supervisor [6].

Accreditation: Procedure by which an accreditation body gives formal recognition that a laboratory or person is competent to carry out specific tasks.

Accreditation body: Independent science-based organization which has the authority to grant accreditation, such as UKAS.

Accuracy (bias, trueness): Ability to get the true result [10]. For quantitative tests the accuracy expresses the closeness of agreement between the true value and the value obtained by applying the test procedure a number of times. It is affected by systematic and random errors.

Accuracy (of a measuring instrument): Ability of a measuring instrument to give responses close to a true value.

Note: In this context accuracy is a qualitative concept. [11] 
Action limit: See Limit.

Administrative review: Procedure used to check for consistency with laboratory policy and for editorial correctness. This review may be performed by the author of the report or other personnel.

Aliquot: Portion of a liquid sample or solution.

Alternative hypothesis: See Hypothesis testing.

Analysis: See Test.

Analysis of variance (ANOVA): Statistical technique which can be used to separate and estimate the different causes of variation [12].

Analyte or Target analyte: Substance to be identified or measured [13].

Surrogate analyte: Well-characterized substance which is taken as representative of the analyte [14].

Analytical batch or run: Complete set of analytical samples with an appropriate number of standards and quality control samples for their validation. Several runs (or batches) may be completed in one day, or one run (or batch) may take several days to complete. See also Batch.

Analytical method: See also Method, Procedure and Test.

Analytical system (measurement system): Complete set of measuring instruments and other equipment assembled to carry out a specified measurement task [5]. In the context of analysing controlled drugs in seized materials or biological specimens, the analytical system consists of the laboratory balance(s), $\mathrm{pH}$ meter, chromatograph, thin layer chromatography equipment, etc. which are used by the analyst to carry out the analysis.

Archive: Collection of documents and records purposefully stored for a defined period of time [15].

Arithmetic mean or Average: Sum of the individual values in a set divided by the number of values [16].

Assay: Quantitative measurement of an analyte.

Assigned value: See Value.

Audit: Independent review conducted to compare the various aspects of the laboratory's performance with a standard for that performance. Also defined as a systematic, independent and documented process for obtaining audit evidence and evaluating it objectively to determine the extent to which audit criteria are fulfilled [6]. 
Auditors: Personnel who carry out an audit. They can be internal, involving suitably trained personnel from within the laboratory's own organization, or external, for example from an accreditation body or other independent organizations.

Horizontal (system) audit: Examines one element in a process on more than one item. It is a detailed check of a particular aspect of the documentation and implementation of the quality management system or examination processes [17]. For example, a horizontal audit might check that document control measures are in place or might check the calibration of pipettes.

Vertical (process) audit: Examines more than one element in a process, on one item. It is a detailed check that all elements associated with a chosen examination (test) are implemented. In any single audit, one or a number of examinations that have recently passed through the laboratory are randomly selected [17]. For example, a group of samples is followed through the complete laboratory process from receipt, to analysis, to reporting.

Audit trail: Detailed records kept of actions carried out by personnel such that subsequent examination of the records by an auditor would allow a sequence of actions to be recreated or understood. Examples of an audit trail include $(a)$ records of which member of staff did what work, when it was done, procedures used, instruments used etc; $(b)$ records of changes made in documents, laboratory procedures, results and reports, who made the changes, when they were made, the reasons why they were made, and who authorized the changes. An electronic audit trail is a recording system built in to a computer-based system which keeps a record of all changes of the types mentioned.

Authorization: Process by which personnel or procedures are approved for the work of the laboratory by suitably qualified staff.

Authorized personnel: Personnel who, because of their seniority and experience, have permission to access controlled areas of the laboratory or else can approve procedures for use in a laboratory.

Average: See Arithmetic mean.

Batch or Analytical batch: Group of one or more samples that are analysed under conditions approaching repeatability. Usually it should contain calibrators and quality control samples in addition to the real samples to be analysed.

Best fit: See Goodness of fit.

Bias: Difference between the expectation of the test result and an accepted reference value. There may be one or more systematic error components contributing to the bias [14]. Bias is the total systematic error as contrasted to random error. A larger systematic difference from the accepted reference value is reflected by a larger bias value [11]. See also Accuracy and Trueness. 


\section{Binomial distribution: See Distribution.}

Biological matrix: Discrete material of biological origin that can be sampled and processed in a reproducible manner. Examples are blood, serum, plasma, urine, faeces, saliva, sputum, and various discrete tissues.

Blank: Specimen not containing the analyte.

Blind specimen or sample: Specimen or sample that is analysed by an operator who is unaware at the time of the analysis that the sample is for control purposes [18].

Blunder: Big mistake, especially one which seems to be the result of carelessness or stupidity [15]. See Outlier.

Calibrations: Set of operations that establish, under specified conditions, the relationship between values indicated by a measuring instrument or measuring system, or values represented by a material measure, and the corresponding known values of a measurand [19].

Calibration curve: Relationship between the signal response of the instrument and various concentrations of analyte in a suitable solvent or matrix [16].

Calibration interval: Frequency of specific performance tests that are made on each instrument or item of equipment as part of the laboratory's preventive maintenance programme [20].

Calibration laboratories: Laboratories that perform calibrations [19, 21].

Calibration method: Defined technical procedure for performing a calibration [19].

Calibration range: See Range.

Calibration record(s): Record(s) usually maintained in instrument or equipment logbooks that contain details of calibrations carried out, dates on which they were performed and the operator(s) involved. Calibrations may be carried out by internal personnel or external organizations. The latter may provide the laboratory with a calibration certificate.

Calibration standard: Biological matrix to which a known amount of analyte has been added or spiked. Calibration standards are used to construct calibration curves from which the concentrations of analytes in quality control and unknown samples are determined. See also Calibrator.

Calibrator: Pure analyte in a suitable solvent or matrix used to prepare the calibration curve. Calibrators are similar in composition to controls but must be prepared separately from them, since controls are used to check on the accuracy of the calibration curve. 
Candidate method: Analytical method which has been selected and developed for a particular analytical problem and which must be validated to show that it is fit for the intended analytical purpose before being used.

Case records: All notes, reports, chain of custody records, charts, analytical data, and any correspondence generated in the laboratory pertaining to a particular case.

Case review: Scrutiny of documents pertaining to a case, usually by a senior member of staff, to ensure that the case has been processed according to the laboratory's specified procedure. The review would include a check on submission forms, chain of custody records, analytical results and interpretations and of the final report.

Certification: Procedure by which a certifying body gives formal recognition that an organization, person, process, service or product complies with given specifications.

Certified reference material (CRM): Reference material one or more of whose property values are certified by a technical procedure, accompanied by or traceable to a certificate or other documentation which is issued by a certifying body [19].

Certifying body: Independent science-based organization which has the competence to grant certifications. The certifying body may or may not be accredited.

Chain of custody/chain of evidence: Procedures and documents that account for the integrity of a specimen or sample by tracking its handling and storage from its point of collection to its final disposition [8].

Check sample: Test sample used to ensure that the performance characteristics of an instrument are being maintained.

\section{Chi-square distribution: See Distribution.}

Chirality: Property of a molecule not to be superimposable on its mirror image. Owing to asymmetry in their structures these molecules can exist as different isomers and will have special optical and biological properties.

\section{Clerical error: See Error.}

Client: Person, group or organization which submits samples to a laboratory for analysis. Clients may be internal, for example, from another department within the laboratory's own organization, or external, for example, another organization.

Cochran test: See Outlier. 
Co-chromatography: Procedure for the identification of an unknown substance by chromatographic comparison with a known substance in which, prior to the chromatographic step(s), the purified test solution is divided into two parts and:

- One part is chromatographed as such;

- The other part has the standard analyte that is to be identified added to it and the resulting solution is then chromatographed. The amount of standard analyte has to be similar to the estimated amount of the analyte in the test solution.

If the unknown substance is the same as the added standard analyte, the chromatographic peaks from the two samples will have the same retention time and shape.

Coefficient of variation or Relative standard deviation: Measure used to compare the dispersion or variation in groups of measurements. It is the ratio of the standard deviation to the mean, multiplied by 100 to convert it to a percentage of the average $[16,20]$.

Collaborative studies: Organization, performance and evaluation of tests on the same or similar items or materials by two or more different laboratories in accordance with predetermined conditions. The main purpose is validation of analytical methods or establishment of reference methods [22].

Competence: Ability to carry out a task correctly. The term takes on additional meaning under ISO/IEC 17025, as laboratory personnel are required to establish and maintain competence in all procedures which are accredited to the standard.

Competency test: Evaluation of a person's ability to perform work in any functional area prior to the performance of independent casework.

Compliance: Demonstrable proof that a laboratory has set up documented procedures to implement all of the requirements of a recognized standard (such as ISO/IEC 17025). If these procedures are verified by an external agency which can certify that they are in place, are used and are effective, then the laboratory is eligible for accreditation.

Concentration: Amount of a substance, expressed in mass or molar units, in a unit volume of fluid or mass of solid.

Confidence interval: Range of values which contains the true value at a given level of probability. This level of probability is called the confidence level.

Confidence level or Confidence coefficient: Measure of probability associated with a confidence interval expressing the probability of the truth of a statement that the interval will include the parameter value [23].

Confidence limits: Extreme values or end values in a confidence interval. See Limit. 
Confirmatory test: Second test by an alternate chemical method for unambiguous identification of a drug or metabolite [10].

Consensus value: See Value.

Contamination: Gain of analyte during the extraction process, in contrast to the losses usually incurred which are assessed by the recovery.

Contract review: Procedures to ensure that the requirements of its clients are adequately defined, documented and understood and that it has the capability of meeting these requirements before agreeing (making a contract) to do the work. This is termed "contract review" in ISO/IEC 17025.

Contractor: Organization that provides a certain service under contractual conditions. It should be ensured that the contractor provides services in line with specified criteria of competence.

Control chart: Graphical plot of test results with respect to time or sequence of measurements, with limits drawn within which results are expected to lie when the analytical scheme is in a state of statistical control [14].

Cusum chart: In the cusum chart each result is compared with a reference, usually the assigned or target value. The differences from the reference are then accumulated, respecting the sign, to give a cumulative sum of differences from the standard. The cusum chart has the advantage of identifying small persistent changes in the analytical scheme faster than the Shewhart chart [16].

Shewhart chart: Chart where the variable of interest is plotted against batch or time. The observed values are compared with the expected or true value. Lines corresponding to the mean value obtained from replicate analysis of reference material and warning and action limits are inserted to provide objective criteria for the interpretation of the chart [14].

\section{Control limit: See Limit.}

Controls: Specimens used to determine the validity of the calibration, that is, the linearity and stability of a quantitative test or determination over time. Controls are either prepared from the reference material (separately from the calibrators, that is, weighed or measured separately), purchased, or obtained from a pool of previously analysed specimens. Where possible, controls should be matrix-matched to specimens and calibrators [8]

Positive control: Control that contains the analyte at a concentration above a specified limit.

Negative control: Control that contains the analyte at a concentration below a specified limit. Usually a drug-free specimen (blank) is used as a negative control. 
Correction for recovery: Recovery of analytes in a method is frequently less than $100 \%$. If there is no internal standard (which automatically compensates for incomplete recovery) then the results of analysis must be multiplied by a correction factor to obtain the values which would have been produced if the recovery had been $100 \%$. This implies that the recovery of the method is known, which will be true if the method has been validated, as recovery is one of the performance characteristics which is measured.

Corrective action: Action taken to eliminate the causes of an existing deviation, defect or other undesirable situation in order to prevent recurrence.

Correlation coefficient: Number showing the degree to which two variables are related. Correlation coefficients range from 0 (no correlation) to -1 or +1 (perfect correlation).

Crime/forensic laboratory: Laboratory which employs one or more full-time scientists whose principal function is the examination of physical evidence for use in legal proceedings and who provide testimony with respect to such physical evidence to the courts.

Cross reactivity: Response (of method) to analogues, metabolites, or other non-target components that may be present in the matrix(es).

Cross-reacting substances: In immunoassays, a substance that reacts with antiserum produced for the target analyte [10].

Cusum chart: See Control chart.

Cut-off concentration: Concentration of a drug in a specimen used to determine whether the specimen is considered positive or negative [24]. In some circumstances it is recommended that the cut-off concentration should be set equal to the limit of detection. See Threshold.

Deficiency: Inadequacy; lacking in some necessary quality or element. Deficiencies include missing data, incomplete data, or incomplete reports.

Degrees of freedom: Number of independent comparisons which can be made between the members of a sample [23].

Detection limit: See Limit.

Deviation: Departure from what is considered normal [15]. See Standard deviation.

Discrepancy: Any reported results which differ from the consensus results. Discrepancies may be classified as administrative, systematic, analytical or interpretive. 
Discrimination: Ability to recognize and understand the differences between two things [15]. Also, the ability of a measuring instrument to respond to small changes in the value of the stimulus.

Discrimination threshold: Smallest change in a stimulus which produces a perceptible change in the response of a measuring instrument. The discrimination threshold may depend on, for example, noise (internal or external), friction, damping, inertia, quantization.

Distribution: A ranking, from lowest to highest, of the values of a variable and the resulting pattern of measures or scores, as these are often plotted on a graph [20]. A frequency distribution, for example, gives the possible values of a parameter versus the number of times each value occurred in the sample or population. In many instances it refers to the spread of the individual values of a sample or population around the mean.

Binomial distribution: Based on the idea that if only one out of two possible outcomes can occur on any one occasion, then the theoretical distribution of the different combinations of outcomes which could occur can be worked out if the number of occasions that had taken place is known. One characteristic of this distribution is that it consists of a limited or finite number of events $n$. When $n$ becomes very large, tending to infinite, the binomial distribution becomes the normal distribution.

Chi-square distribution: This distribution may be considered as that of the sum of squares of $v$ independent normal variates in standard form. The parameter $v$ is known as the number of degrees of freedom [23].

$\boldsymbol{F}$-distribution: Theoretical distribution used to study population variances. It is the distribution of the ratio of two independent variables each of which has been divided by its degrees of freedom [20].

Normal distribution: Purely theoretical continuous probability distribution in which the horizontal axis represents all possible values of a variable and the vertical axis represents the probability of those values occurring. The scores on the variable are clustered around the mean in a symmetrical, unimodal pattern known as the bell-shaped curve or normal curve. In a normal distribution, the mean, median, and mode are all the same [20]. The normal distribution is obtained when the number of events in the binomial distribution, $n$, becomes very large, tending to infinite.

Probability distribution: Distribution giving the probability of a value of $x$ as a function of $x$, or more generally, the probability of joint occurrence of a set of variates $x_{1} \ldots x_{p}$ as a function of those quantities [23].

t-distribution: Theoretical probability distribution used in hypothesis testing. As with the normal distribution, the $t$-distribution is unimodal, symmetrical, and bell-shaped [20]. 
Theoretical probability distribution: Number of times we would expect to get a particular number of successes in a large number of trials [20]. Important theoretical probability distributions are the normal, $t$, chi-square and $F$-distributions.

$z$-distribution: Normal distribution in which the scores are the $z$-scores [20].

Distribution function: The distribution function $F(x)$ of a variate $x$ is the total frequency of members with variate values less than or equal to $x$. As a general rule, the total frequency is taken to be unity, in which case the distribution function is the proportion of members bearing values less than or equal to $x$ [23].

Dixon test: See Outlier.

Document control: Mechanism by which quality management system documents are created and amended, reviewed, approved, distributed and archived to ensure that all staff use the latest authorized versions.

Double blind procedure: Means of reducing bias in an experiment, for example in the clinical context, by ensuring that both those who administer a treatment and those who receive it do not know (are blind to) which subjects are in the control and experimental group, that is, who is and is not receiving the treatment [20].

Duplicate samples or specimens: Two aliquots of a sample or specimen analysed at the same time.

Dynamic range: Range over which a relationship exists between analyte concentration and assay response [25].

End determination or End-step determination: Final step in a sequence of stages comprising an analytical method, usually involving the application of a technique to an extract or other sample preparation to produce data on the composition of the sample.

ENFSI: European Network of Forensic Science Institutes.

Equipment: In general, the apparatus required for any operation [26]. More specifically, the analytical measurement hardware, for example a gas chromatograph.

Error: Something done which is considered to be incorrect or wrong [15].

Absolute error: Difference between the analytical result and the true value [27].

Clerical error: Mistake made during routine jobs that are done in an office or laboratory e.g. transcription errors, specimen misidentification, filing error.

Maximum tolerable error: Extreme values of an error permitted by specifications, regulations, etc. for a given determination. [28]

Random error: Component of the total error of a measurement which varies in an unpredictable way. This causes the individual results to fall on both sides of 
the average value $[5,12]$. Note: Random error is equal to error minus systematic error. Because only a finite number of measurements can be made, it is possible to determine only an estimate of random error.

Relative error: Absolute error of a measurement divided by the assigned value of the analyte [28]. See Coefficient of variation and Relative standard deviation.

Systematic error: Component of the total error of a measurement which varies in a constant way . This causes all the results to be in error in the same sense $[12,28]$. Mean that would result from an infinite number of measurements of the same measurand carried out under repeatability conditions minus a true value of the measurand. Note: Systematic error is equal to error minus random error. Like true value, systematic error and its causes cannot be known.

Total error: Sum of random and systematic errors.

Type I error: Error made by wrongly rejecting a true null hypothesis.. If the null hypothesis is that the sample should be negative, a type I error will generate a false positive result [20].

Type II error: Error made by wrongly accepting a false null hypothesis [20]. If the null hypothesis is that the sample should be negative, a type II error will generate a false negative result.

Estimate value: See Value.

Evaluation: Systematic examination of the extent to which a product, process or service fulfils specified requirements [22].

\section{Exemplar: See Known standard.}

Expert witness: Appropriately qualified/experienced person, for example, a forensic scientist, familiar with the testing, evaluation and interpretation of test results. An expert witness is recognized by the court to provide opinion evidence [29].

Expiration date: Date after which the specified characteristics of a reagent, solution, specimen, control, etc. can no longer be guaranteed.

External standard: Standard prepared directly from a reference substance, for example as a stock solution or serial dilutions of the stock solution. It is not prepared in the same type of matrix as the specimens or samples for analysis and therefore there is no requirement for an extraction step prior to analysis.

False negative: Test result which states that no drug or metabolite is present when, in fact, such a drug or metabolite is present in an amount greater than a threshold or a designated cut-off concentration [30]. 
False positive: Test result which states that a drug or metabolite is present when, in fact, such a drug or metabolite is not present or is present in an amount less than a threshold or a designated cut-off concentration [30].

False positive or False negative rate: A measure of reliability of a method which indicates the percentage of false negative and false positive results. The numbers of true positives/negatives are obtained using a reference method and compared with the numbers of positives/negatives obtained from the test method using the following equations:

False positive rate $(\%)=$ false positives $\times 100 /$ true negatives

False negative rate $(\%)=$ false negatives $\times 100 /$ total known positives

In the absence of a reference method, negative and positive fortified samples must be analysed.

F-distribution: See distribution.

Fitness for purpose: Degree to which data produced by a measurement process enables a user to make technically and administratively correct decisions for a stated purpose [11].

Fit for purpose: A laboratory method that is fit for purpose satisfies the needs of the client and produces results which can be used for their intended purpose, such as within a legal process.

F-test: See Significance test.

Geometric mean: See Mean.

Good laboratory practice (GLP): Organizational process and conditions under which laboratory studies are planned, performed, monitored, recorded and reported. Includes a system of protocols (standard operating procedures) recommended to be followed so as to avoid the production of unreliable and erroneous data [31, 32].

Goodness-of-fit: How well a model, a theoretical distribution, or an equation matches actual data [20].

Grubbs test: See Outlier.

Harmonization: Bringing about agreement on terminology, concepts, etc. so that different entities can interact based on the same terms of reference.

Hypothesis test: See Significance test.

Hypothesis testing or Significance testing: Process of assessing the statistical significance of findings. It involves comparing empirically observed sample findings with theoretically expected findings, expected if the null hypothesis is true (see Significance test). 
This comparison allows one to compute the probability that the observed outcomes could have been due to chance alone [33]. See Non-parametric test.

Alternative hypothesis: Hypothesis that must be accepted if the null hypothesis is rejected [33].

Null hypothesis $\left(\mathbf{H}_{\mathbf{o}}\right)$ : Any hypothesis to be tested. The term null implies that there is no difference between the observed and known values other than that which can be attributed to random variation $[12,33]$.

IEC: International Electrotechnical Commission.

ILAC: International Laboratory Accreditation Cooperation, which publishes relevant documents such as the ILAC recommendations G19:2002.

Imprecision: See Precision.

Independent test result: Result obtained in a manner not influenced by any previous results on the same or similar material [14].

Influence quantity: Quantity which is not the subject of measurement but which influences the result, e.g. environmental conditions [28].

In-house reference material: See Reference material.

Initial test: See Screening test.

Instrument (instrumentation, measuring instrument): Device intended to make a measurement, alone or in conjunction with other equipment.

Instrument linearity: Straight-line relationship between analyte concentrations and the instrument response, in which a change in concentration causes a proportional change in response [30].

Instrument validation: Process of establishing that an instrument at any given moment is able to perform according to its design specification This process might be achieved for example by means of calibration or performance checks.

Interference study: Study to check the selectivity (or specificity) of a method by adding materials which might be encountered in specimens and which it is suspected might cause interference.

Interfering substance: Substances other than the analyte that give a similar analytical response or alter the analytical result [10].

Interlaboratory studies: See Collaborative studies. 
Interlaboratory test comparisons: See Collaborative studies.

Intermediate precision: See Precision (intermediate).

Internal auditor: See Audit.

Internal standard: Compound added to a sample in known concentration to facilitate the qualitative identification and/or quantitative determination of the sample components [11]. The physicochemical characteristics of the internal standard should be as close as possible to those of the analyte, for example, a structurally similar analogue or a stable isotope-labelled analogue of the analyte can be used.

International standard: Standard recognized by an international agreement to serve internationally as the basis for fixing the value of all other standards of the quantity concerned [29].

Interpretation: Explanation of what analytical results mean based on chemical, pharmacological, toxicological and statistical principles.

Intralaboratory test comparisons: Organization, performance and evaluation of tests on the same or similar items or materials within the same laboratory in accordance with predetermined conditions [22].

ISO: International Organization for Standardization [34]: An organization which defines standards in a diverse range of fields. Relevant standards amongst these are the following:

ISO/IEC 17025:2005: General Requirements for Competence of Testing and Calibration Laboratories: this provides a quality standard for testing and calibration laboratories. The associated ILAC (International Laboratory Accreditation Conference) recommendations G19:2002 (Guidelines for Forensic Science Laboratories) interpret ISO 17025 specifically for forensic science laboratories.

ISO 9001:2000 and ISO 9001:2008: Quality Management Systems-Requirements, an older standard, dealing primarily with management structure of an organization.

ISO/IEC 17020:1998: General criteria for the operation of various types of bodies performing inspection (this standard is used for accreditation of crime scene investigation);

ISO/IEC 17043: Conformity assessment-General requirements for proficiency testing [35].

ISO/IEC Guide 43-1:1997: Proficiency testing by interlaboratory comparisonsPart 1: Development and operation of proficiency testing schemes.

ISO/IEC Guide 43-2:1997: Proficiency testing by interlaboratory comparisonsPart 2: Selection and use of proficiency testing schemes by laboratory accreditation bodies. 
Known standard: Specimen of an identified source acquired for the purpose of comparison with an evidence sample; synonymous with exemplar.

Laboratory: Facilities where analyses are performed by qualified personal and with adequate equipment.

Laboratory Information Management System: See LIMS.

Laboratory manager: Qualified individual who assumes professional, organizational, educational and administrative responsibility for the laboratory's drug testing.

Least-squares: Statistical method of determining a regression equation, that is, the equation that best represents the relationship among the variables [20].

Level of significance: Probability that a result would be produced by chance alone, i.e. the probability of incorrectly rejecting the null hypothesis. It is therefore the probability of making a type I error.

Limit: Prescribed or specified maximum or minimum amount, quantity or number [36]

Action limit: Corresponds to \pm 3 standard deviation from the mean. If an observed value falls outside the action limit then immediate measures must be taken to identify the cause and to take remedial action.

Confidence limit: The limits of the confidence interval.

Control limit: The limits, on a control chart, that are used as criteria for action or for judging whether a set of data does or does not indicate lack of statistical control.

Detection limit: See Limit of detection.

Quantitation limit: The smallest measured content from which it is possible to quantitate the analyte with an acceptable level of accuracy and precision.

Warning limit: Corresponds to \pm 2 standard deviations from the mean. Even if the method is under statistical control approximately $5 \%$ of results may be expected to fall outside the warning limits.

Limit of detection (LOD): Smallest measured content from which it is possible to deduce the presence of the analyte with reasonable statistical certainty [5, 37]. Alternatively, LOD is the lowest concentration of an analyte that the analytical procedure can reliably differentiate from background noise or LOD is the smallest amount or concentration that can be readily distinguished from zero and be positively identified according to predetermined criteria and/or levels of confidence [38].

The limit of detection, expressed as the concentration $c_{L}$, or the quantity $q_{L}$, is derived from the smallest measure $x_{L}$, that can be detected with reasonable certainty for a given 
analytical procedure. The value of $x_{L}$ is given by the equation:

$$
x_{L}=x_{b l}+k s_{b l}
$$

where $\mathrm{x}_{b l}$ is the mean of the blank measures and $s_{b l}$ the standard deviation of the blank measures, and $k$ is a numerical factor chosen according to the confidence level desired [39].

\section{Limit of quantitation or quantification (LOQ)/Lower limit of quantification} (LLOQ): Smallest measured content from which it is possible to quantify the analyte with an acceptable level of accuracy and precision. In some laboratories the $L L O Q$ is termed the lowest calibration concentration of the working range, as accuracy and precision of this concentration is verified in every analytical run/batch. Usually this is synonymous with Limit of quantification but in principle it should be specified as a calibration range has both a lower and an upper limit.

The ability to quantify is generally expressed in terms of the signal or analyte (true) value that will produce estimates having a specified relative standard deviation (RSD), commonly $10 \%$.

Thus: $\mathrm{L}_{\mathrm{Q}}=\mathrm{k}_{\mathrm{Q}} \sigma_{\mathrm{Q}}$

where $\mathrm{L}_{\mathrm{Q}}$ is the Quantification Limit, $\sigma_{\mathrm{Q}}$ is the standard deviation at that point, and $\mathrm{k}_{\mathrm{Q}}$ is the multiplier whose reciprocal equals the selected quantifying RSD. The IUPAC default value for $\mathrm{k}_{\mathrm{Q}}$ is 10 [11].

Limiting mean: Asymptotic value or population mean of the distribution that characterizes the measured quantity; the value that is approached as the number of observations approaches infinity [11].

LIMS (Laboratory Information Management System): Software package for collating, calculating, controlling and disseminating analytical data. It can perform a variety of functions, from specimen registration and tracking; to processing captured data, quality control, financial control and report generation [40].

Linear regression: Method of describing the relationship between two or more variables by calculating a "best fitting" straight line or graph [20].

Linearity: Ability of an analytical method to elicit test results that are directly, or by means of well-defined mathematical transformations, proportional to the concentration of analytes in samples within a given range. The ability of the method to obtain test results proportional to the concentration of the analyte. The linear range is by inference the range of analyte concentrations over which the method gives test results proportional to the concentration of the analyte.

Logbook: Book which records laboratory activities, e.g. instrumentation, maintenance of instrumentation, sample preparation and reagents. 
Maintenance: Activity of keeping something such as facilities, machines or instrumentation in good condition by regularly checking it and doing necessary repairs [15].

Management review: Scheduled review of the laboratory's quality management system and its testing activities organized by the quality manager to ensure that they remain suitable and effective or to identify any changes and improvements required. The review should be carried out by high-level management of the organization of which the laboratory is a part, the quality manager and other relevant staff [1].

Management structure: Hierarchy of personnel in an organization. This is often portrayed in an organigram or tree diagram.

Matrix: Material which contains the analyte e.g. urine, blood etc.

Matrix effect: Direct or indirect alteration or interference in response due to the presence of unintended analytes (for analysis) or other interfering substances in the samples.

Maximum tolerable error: See Error.

Mean: When not otherwise specified, refers to arithmetic mean .

Geometric mean: The $n$th root of the product of $n$ individual values.

Measurand: Particular quantity subject to measurement. Note: Specification of a measurand may require statements about quantities such as time, temperature and pressure.

Measurement: Set of operations having the object of determining a value of a quantity [32].

Measurement procedure: Set of operations, described specifically, used in the performance of measurements according to a given method [32]. Note: a measurement procedure is normally recorded in a document that is sometimes itself a measurement procedure or measurement method and is usually in sufficient detail to enable the operator to carry out a measurement without additional information.

Measurement system: See Analytical system.

Measuring instrument:* See Instrument.

Median: Middle value of a ranked set of data [16].

Metabolite: Compound produced in the body as a result of biochemical processes. 
Method (or Analytical method): Detailed (defined) procedure of a technical operation for performing an analysis. See also Test procedure.

Method authorization form: Document which certifies that an analytical method has been validated for its intended purpose in the laboratory and has been authorized for that purpose by the laboratory manager, who should sign the form.

Method of measurement: Logical sequence of operations, described generically, used in the performance of measurements [32].

Method scope: Circumstances or conditions under which a method will produce valid analytical results. This statement is usually contained in the standard operating procedure for the method and will refer to the analytes included, sample matrix type(s), concentration range, known interferences, etc.

Method traceability: Property of the result of a measurement or the value of a standard whereby it can be related, with a stated uncertainty, to stated references, usually national or international standards, through an unbroken chain of comparisons [14].

Method validation: Confirmation by examination and provision of objective evidence that the particular requirements for a specific intended use of a method are fulfilled [41]. Also defined as the process of verifying that a method is fit for purpose, i.e. for the use of solving a particular analytical problem or as the process by which it is established, by laboratory studies, that the performance characteristics of the method meet the requirements for the intended analytical application. A working definition may include the ideas that a valid method [42]:

- Is suitable (reliable) for its purpose;

- Provides useful analytical data in a specific situation;

- Meets the pre-determined requirements (specifications) of the analytical problem;

- Has an established level of performance (accuracy, consistency, reliability);

- Does what it is supposed to do.

Mode (Statistic): Value or values occurring most frequently in a set of data [16].

National standard: Standard recognized by an official national decision as the basis for fixing the value, in a country, of all other standards of the quantity concerned.

Negative: Indicates that the analyte is absent or below a designated cut-off. "Not detected" is sometimes used as a synonym of negative although this is not recommended.

Negative control: See Control. 
Negative predictive value: Number of negative results obtained by new method divided by number of true negative results.

Non-conformance (non-conformity): When the work carried out by the laboratory is inconsistent with its Quality Management System (for example, the work deviates from an operating procedure) or the requirements of its clients, this is termed a "non-conformance" in ISO 17025.

None detected: Indicates the absence of an analyte within the specifications of the test(s) performed.

Non-parametric test: Statistical method that makes no assumptions about the shape of the distribution of the population from which the sample data are taken [12].

One-tail test: Hypothesis test stated so that the chances of making a Type I error are located entirely in one tail of a probability distribution, e.g. if we wish to test only whether method A is more precise than method B and not whether method B is more precise than method A [20].

Two-tail test: Statistical test in which the critical region (region of rejection of the null hypothesis) is divided into two areas at the tails of the sampling distribution, e.g. if we wish to test whether method A and B differ in their precision [20].

\section{Normal distribution: See Distribution.}

Not detected: Use of this term as a synonym for negative is not recommended.

Notes: Documentation of procedures, standards, controls and instruments used, observations made, results of tests performed, charts, graphs, photos, and other documents generated which are used to support the examiner's conclusions.

Null hypothesis: See Hypothesis testing.

One point calibration: Simplified calibration procedure using a single calibrator and a blank.

One-tail test: See Non-parametric test.

Organization: Companies, corporations or institutes (or part of e.g. laboratory) private or public, that has its own functions and administration. Some of the international organizations dealing with quality assurance $(Q A)$ are: The International Association of Forensic Toxicologists (TIAFT), The International Federation of Clinical Chemistry (IFCC), the International Olympic Committee (IOC), The International Organization for Standardization (ISO), The International Programme on Chemical Safety (IPCS), The International Union of Pure and Applied Chemistry (IUPAC) and the Organisation for Economic Cooperation and Development (OECD). 
Organigram: Diagram showing the organizational structure of a business, laboratory or other enterprise, such as the one shown below. See also Management structure.

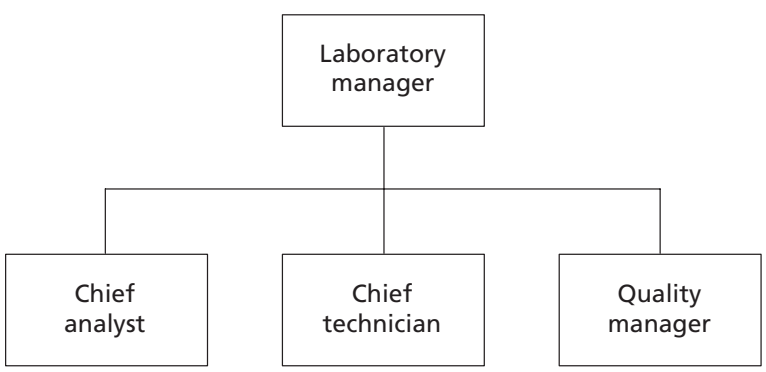

Outlier: Result that appears to differ unreasonably from the population of the other results. Tests for outliers include:

Cochran test: This compares the largest of a set of variances with the other variances in the set [43].

Dixon test: This compares the difference between a measurement and the one nearest to the measurement in size with the difference between the highest and lowest measurements in the set [12].

Grubbs test: Test for outliers which is now recommended to replace the Dixon test and to be used sequentially after the Dixon test [44]. The single Grubbs test statistic is calculated as the percentage decrease in the standard deviation of a set of results following the removal of either the highest or lowest value in the set, whichever gives the largest decrease in the standard deviation. The pair Grubbs statistic is calculated in an analogous manner by removing the two highest, two lowest or else both the highest and the lowest values in the original set of results, whichever gives the lowest standard deviation. The presence of an outlier or a Grubbs outlier pair is indicated if the Grubbs statistic exceeds a critical value, which depends on the number of results in the set and which is given by a reference table $[44,45]$.

Parametric test: Statistical techniques designed for use when data have certain characteristics, usually when they approximate a normal distribution and are measurable [20].

Peer: Individual having similar expertise in a relevant and specific functional area gained through documented training and expertise.

Peer review: Review of casework for technical correctness by a peer. See also Technical review.

Performance characteristics: Key aspects of an analytical method which are evaluated for the purposes of method development and validation, including accuracy 
(bias), linearity, limit of detection, limit of quantitation, range, recovery, repeatability, reproducibility, ruggedness, and specificity (selectivity) [46].

Performance qualification: See Performance verification.

Performance specifications: These usually relate to the specifications of an instrument used in the laboratory with respect to sensitivity, reproducibility, etc.

Performance verification (or performance qualification): Formal and nationally traceable method of evaluating the performance of an instrument against previously defined procedures and specifications. Performance verification should involve the use of tests which are not method-specific and which use nationally-traceable calibrators and standards.

Personnel: Persons qualified, (at different levels, and sufficient in number) with adequate training and experience to carry out their assigned functions [19].

Pharmacology: Study of the interactions of drugs with living systems [47].

Plot: Representation of data on or by a graph.

Population (or universe): (Theoretical) entity defined as an entire group of people, things, or events that have at least one trait in common [16].

Population statistics: Statistical descriptors of the population, e.g. mean, median, mode, standard deviation, etc.

Positive: Indicates that the analyte is present at a level above a designated cut-off concentration.

Positive control: See Control.

Positive predictive value: Number of positive results obtained by a new method divided by number of true positive results.

Power of test: Probability of rejecting the null hypothesis when it is false.

Practicability: Ability to put something into practice. In the laboratory, this means the absence of unnecessarily sophisticated equipment, reagents, instruments, or environmental conditions, so that a method is suitable for routine use. [48]

Precision: Closeness of agreement between independent test results obtained under prescribed conditions [14]. It is generally dependent on analyte concentration, and this dependence should be determined and documented [40]. The measure of precision is usually expressed in terms of imprecision and computed as a standard deviation of the test results. Higher imprecision is reflected by a larger standard deviation. Independent test results means results obtained in a manner not influenced by any previous results 
on the same or similar material [14]. Precision covers repeatability and reproducibility [19]. Alternatively, precision is a measure for the reproducibility of measurements within a set, that is, of the scatter or dispersion of a set about its central value.

Precision depends only on the distribution of random errors and does not relate to the true value or specified value.

Precision (intermediate): Precision measured in-between repeatability and reproducibility conditions: for example precision measured between different analysts, over extended timescales, within a single laboratory. Alternative definition: Expresses withinlaboratory variation: different days, different analysts, different equipment, etc.

Presumptive: This is used to describe things that are based on presumptions about what is probably true, rather than on certainty [15].

Presumptive positive: Specimen or sample that has been flagged as positive by screening but which has not yet been confirmed by an adequately sensitive alternate chemical method [10].

Presumptive negative: Specimen or sample that has been flagged as negative by screening. Usually no further tests are carried out and therefore there is no certainty about its content.

Presumptive test: See Screening test.

Preventive (preventative) action: Action taken to eliminate the causes of a potential deviation or other undesirable situation in order to prevent its occurrence [4].

Primary standard: Standard which has the highest metrological qualities in a specified field.

Probability: Mathematical measurement of how likely it is that something will happen, expressed as a fraction or percentage [30]. Values for statistical probability range from 1 or $100 \%$ (always) to 0 or $0 \%$ (never) [20]. The relative frequency obtained after a long run of measurements or results will give good approximations to the true probability [33]. Also, it is a basic concept which may be taken either as indefinable, expressing in some way a "degree of belief", or as the limiting frequency of an occurrence in an infinite random series [49].

Probability distribution: See Distribution.

Probability function: Function of a discrete variate that gives the probability that a specified value will occur.

Procedure: Specified way to perform an activity or process. For quality assurance purposes the procedures should be written [4]. 
Test procedure: Total operation necessary to perform the analysis e.g. preparation of the specimen or sample, of the reference materials or of the reagents, the use of instruments and of formulas for the calculations (when the test is quantitative), the preparation and use of calibration curves and the determination of the number of replicates.

Processed data: Information obtained from raw data in other to make it clear or more readily usable.

Proficiency testing: Ongoing process in which a series of proficiency specimens, the characteristics of which are not known to the participants, are sent to each laboratory on a regular basis. The laboratory is tested on its accuracy in identifying the presence (or concentration) of drug(s) present using its usual procedures. An accreditation body may specify participation in a particular proficiency testing scheme as a requirement of accreditation.

Proficiency testing scheme: Organized system for proficiency testing. A list of schemes in Europe (EPTIS) has been published [50].

Proficiency tests: Tests to evaluate the competence of analysts and the quality performance of a laboratory. In open tests, the analysts are aware that they are being tested; in blind tests, they are not aware. Internal proficiency tests are conducted by the laboratory staff; external proficiency tests are conducted by an agency independent of the analysts or laboratory being tested.

Protocol: Directive listing the procedures to be followed in performing a particular laboratory examination or operation - the overall plan analysis of a particular type of evidence.

Qualitative test: Test that determines the presence or absence of specific drugs or metabolites in the specimen or sample [30].

Quality: Totality of features and characteristics of a product or service that bear on its ability to satisfy stated or implied needs.

Quality assessment: Overall system of monitoring activities which provides assurance that the quality control is performed effectively.

Quality assurance (QA): System of activities whose purpose is to provide to the producer or user of a product or a service the assurance that it meets defined standards of quality with a stated level of confidence [51].

Quality assurance management: All activities of the overall management function that determine and implement quality policy, objectives and responsibilities (adapted from [4]). 
Quality assurance manager: Individual (however named) designated by top management who has the defined authority and obligation to ensure that the requirements of the quality assurance system are implemented and maintained.

Quality assurance programme: Internal control system designed to ascertain that the studies are in compliance with the principles of good laboratory practices (GLP) [32].

Quality audit: Systematic and independent examination to determine whether quality activities and related results comply with planned arrangements and whether these arrangements are implemented effectively and are suitable to achieve the objectives [4].

Quality control: Overall system of activities whose purpose is to control the quality of a product or service so that it meets the needs of users. The aim is to provide quality that is satisfactory, adequate, dependable and economic [51].

External quality control: See Proficiency testing.

Internal quality control: Set of procedures undertaken by a laboratory for continuous monitoring of operations and results in order to decide whether the results are reliable enough to be released. Quality control of analytical data primarily monitors the batchwise trueness of results on quality control materials, and precision on independent replicate analysis of test materials [14] .

Quality management: That aspect of the overall management function that determines and implements the quality policy [4].

Quality management system (QMS): Documentation of a laboratory's policies, systems, procedures and instructions to the extent necessary to assure the quality of its results, to meet relevant jurisdictional, regulatory and safety requirements and to satisfy the needs of the clients. It covers the overall activities of the laboratory, including sampling, analysis and reporting, whether these are within the main laboratory facility itself, mobile/temporary facilities, or external locations such as a clandestine laboratory, the roadside, or the locus of a large drug seizure.

Quality manager: see Quality assurance manager.

Quality manual: Document stating the general quality policies, procedures and practices of an organization [52].

Quality policy: Statement by top management regarding the laboratory's adherence to principles of quality. It may set forth codes of practice or ethics.

Quality standard: Typically, ISO17025, which is the quality standard specifically oriented towards laboratories undertaking chemical analysis. The standard lists a set of requirements which must be fulfilled in order for a laboratory to be accredited to the standard by an external accreditation body. See also ISO. 
Quality system: Organizational structure, responsibilities, procedures, processes and resources for implementing quality management [4]. In a laboratory it refers to the total features and activities of a laboratory aimed at producing accurate work and a high quality product [16]. See Quality management system.

Quantitation (quantification) range: Range of concentrations, including $U L O Q$ and LLOQ, that can be reliably and reproducibly quantified with accuracy and precision through the use of a concentration-response relationship (see also Range)

Quantitation limit: See Limit.

Quantitative test: Test to determine the quantity of drug or metabolite present in a specimen [30].

\section{Random error: See Error.}

Random sample: Sample taken in such a way that all the members of the population have an equal chance of being included, that is, each is chosen entirely by chance [12].

Range (working range, calibration range): Concentration interval for which acceptable accuracy and precision can be achieved [24]. Statistically it is the difference between the minimum and the maximum values of a set of measurements [16].

Raw data: Data that are in their original state and have not being processed [20].

Record: Document which furnishes objective evidence of activities performed or results achieved [4].

Quality records: Audit reports, proficiency tests, customer feedback, corrective and preventative actions, management reviews, etc.

Technical records: All materials relating to cases including sample submission forms, chain of custody documents, case notes including drawings and diagrams, photographs, telephone conversations, spectra, calibration and other quality control data, instrumental operating parameters and print-outs, reports, statements, etc, instrument maintenance records and staff training, competency and authorization records. Technical records should be made at the time the work is done.

Recovery: Percentage of the drug, metabolite, or internal standard originally in the specimen or sample that reaches the end of the procedure [53]. It is measured by adding a known amount of analyte to a blank matrix and comparing this with the amount measured as present by analysis.

Reference material: Material or substance one or more properties of which are sufficiently well established to be used for the calibration of an apparatus, assessing a measurement method or assigning values to materials [19]. 
In-house reference material: Material whose composition has been established by the user laboratory by several means, by a reference method or in collaboration with other laboratories [14].

Reference method or Standard consensus method: Method developed by organizations or groups that use collaborative studies or similar approaches to validate them. Its value depends on the authority of the organizations which sponsor it [16].

Reference preparation: Processed reference material.

Reference standard: Standard, generally of the highest quality available at a given location, from which measurements made at that location are derived [19].

Regression analysis: Method of explaining or predicting the variability of a dependent variable using information about one or more independent variables. Also, techniques for establishing regression equations [20].

Regression curve: Curve that comes closest to approximating a distribution of points in a scatter diagram [20].

Relative error: See Error.

Relative frequency: Number calculated by dividing the number of values with a certain characteristic by the total number of values [20]. It is also the frequency of an event which would occur in the long run given the probability of the event [33].

Relative standard deviation: See Coefficient of variation.

Reliability: Extent to which an experiment, test, or measuring procedure yields accurate results in repeated trials [36].

Repeatability: Closeness of the agreement between the results of successive measurements of the same analyte made under repeatable conditions, e.g. same method, same material, same operator, same laboratory, narrow time period [28, 40]. Results should be expressed in terms of the repeatability standard deviation, repeatability coefficient of variation, or the confidence interval of the mean value. Alternatively, repeatability is the closeness of the agreement between the results of successive measurements of the same measurand carried out under the same conditions of measurement.

Repeatability (of a measuring instrument): Ability of a measuring instrument to provide closely similar indications for repeated applications of the same measurand under the same conditions of measurement.

Replicability: See Replicate analysis.

Replicate analysis: Multiple analysis of separate portions of a test material using the same test method under the same conditions e.g. same operator, same apparatus, same laboratory [29]. 
Report: Document containing a formal statement of results of tests carried out by a laboratory. It should include the information necessary for the interpretation of the test results [19].

Representative sample: Statistically, a sample that is similar to the population from which it was drawn. When a sample is representative, it can be used to make inferences about the population. The most effective way to get a representative sample is to use random methods to draw it [20]. Analytically it is a specimen which is a portion of the original material selected in such a way that it is possible to relate the analytical results obtained from it to the properties of the original material [40].

Reproducibility (within-laboratory): Closeness of the agreement between the results of successive measurements of the same analyte in identical material made by the same method under different conditions, e.g. different operators, different laboratories, long time period [28,40]. Results should be expressed in terms of the reproducibility standard deviation; the reproducibility coefficient of variation, or the confidence interval of the mean value.

Reproducibility limit "R": Value less than or equal to which the absolute difference between two test results obtained under reproducibility conditions may be expected to be with a probability of $95 \%$.

Resolution: Ability to distinguish meaningfully between closely adjacent values [28].

Response time: Time interval between the instant when a stimulus is subjected to specified abrupt change and the instant when the response reaches and remains within specified limits of its final steady value [11].

Result: Information obtained from a test or series of tests. Usually it refers to processed data.

Result of a measurement: Value attributed to a measurand, obtained by measurement. When the term is used, it should be made clear whether it refers to the indication, the uncorrected result, the corrected result, and whether several values are averaged. A complete statement of the result of a measurement includes information about the uncertainty of measurement.

Retention sample or Specimen: Amount of material equivalent in quantity to the assay specimen and taken from the consignment in a manner similar to that used to assay the sample. It should be kept stored under specified conditions [54].

Review: Evaluation of laboratory results to ensure that they have been correctly interpreted. See also Management review. 
Robustness or Ruggedness: Capacity of a test to remain unaffected by small but deliberate variations in the procedures. It is measured by deliberately introducing small changes to the method and examining the consequences [40]. See Influence quantity. Ruggedness of an analytical method is alternatively defined as the degree of reproducibility of test results obtained by the analysis of the same samples under a variety of conditions, such as different laboratories, analysts, instruments, lots of reagents, elapsed assay times, assay temperatures or days. Ruggedness is normally expressed as the lack of influence on the test results of operational and environmental variables of the analytical method. Ruggedness is a measure of reproducibility of test results under the variation in conditions normally expected from laboratory to laboratory and from analyst to analyst.

Ruggedness test: Intralaboratory experimental plan, used before undertaking an interlaboratory study, to examine the behaviour of an analytical process when small changes in the environmental and/or operating conditions are made, akin to those likely to arise in different laboratories.

\section{Run sequence: See Analytical run or Batch.}

Safety manager: Individual (however named) designated by top management who, irrespective of other responsibilities, has the defined authority and obligation to ensure that the requirements of the safety system are implemented and maintained.

Safety manual: Document stating the safety policy and describing the various elements of the safety system of an organization.

Sample: Analytically (equivalent to specimen), it is a representative portion of the whole material to be tested. Statistically, it is a set of data obtained from a population [16].

Sample statistics: Statistical descriptors of the sample, e.g. mean, median, mode, standard deviation, range or size.

Sampling: Analytically, it is the whole set of operations needed to obtain a sample or specimen including planning, collecting, recording, labelling, sealing, shipping, etc. Statistically it is the process of determining properties of the whole population by collecting and analysing data from a representative segment of it [36].

Sampling plan: Procedure for taking samples of seized materials and biological specimens, both at the scene and within the laboratory.

Scatter diagram or scatter plot or scattergram: Pattern of points that results from plotting two variables on a graph. Each point or dot represents one subject or unit of analysis and is formed by the intersection of the values of the two variables [20].

Scope of a method: See Method scope. 
Screening test or Initial test or Presumptive test): First test carried out on a specimen for the purpose of determining a presumption of a positive or negative assay. Usually presumptive positives are followed by a confirmatory test.

Selectivity: Extent to which a method can determine particular analyte(s) in a complex mixture without interference from the other components in the mixture [40]. A method that is perfectly selective for an analyte or group of analytes is said to be specific.

Sensitivity: (a) Difference in analyte concentration corresponding to the smallest detectable difference in the response of the method. It is represented by the slope of the calibration curve. Sometimes it is used, erroneously, to mean detection limit.

(b) Incidence of true positive results obtained when a test is applied to samples known to contain the analyte [55].

(c) Change in the response of a measuring instrument divided by the corresponding change in the stimulus. The stimulus may, for example, be the amount of the measurand present.

Shewhart chart: See Control chart.

Significance test (Hypothesis test): Statistical test whose purpose is to draw a conclusion about a population using data from a sample. It is used to determine the likelihood that observed characteristics of samples have occurred by chance alone in the population from which the samples were selected. Frequently used significance tests include the following:

Comparison-of-means test or $\boldsymbol{t}$-test: Compares the mean of the results from one sample taken from a given population with the mean of the results from a second sample taken from the same population, with the two sets of results having, for example, been produced by different analytical methods. It answers the question, "Are the two means significantly different?" The null hypothesis is that the means are not significantly different and that the samples are therefore part of the same population. It is assumed that the variances of the samples are the same and that the samples are representative of the whole population. The larger the number of results for each sample, the more likely this is to be true. Statistical comparison of the means will indicate whether any differences between the samples could have arisen by chance alone. The $t$-test is used under particular circumstances, for example, when the size of the samples is small (usually less than 20) or when a single sample is taken from a population for which the variance is unknown.

Variance-ratio test or $\boldsymbol{F}$-test: In the comparison-of-means test it is assumed that the variance of each sample is the same. A variance-ratio test is used to check if this assumption is reasonable. 
Significant figures: Number of figures that are consistent with the precision of the test.

Skewness: Said of measures or scores that are bunched on one side of a central tendency parameter (mean, median, mode) and trail out on the other. The more skewness in a distribution, the more variability in the scores [20]. Also used to refer to asymmetry in, for example, a chromatographic peak shape ("tailing" and "fronting").

Specification: Statement of requirements, usually in written form.

Specificity: (a) See Selectivity.

(b) Incidence of true negative results obtained when a test is applied to samples known not to contain the analyte.

Specimen: Analytically, equivalent to sample. In the context of this Glossary, any biological material for examination, study or analysis..

Spiked sample: A test material containing a known addition of analyte [14].

Split-level model: Statistical model that splits the study sample according to a predetermined assumption so that only a portion of the cases falls into the category of interest, e.g. so that only some of the specimens that were positive for group A of drugs will be positive for group $\mathrm{B}$.

Split specimen or Sample: Practice of dividing a specimen or sample. A urine specimen, for example, may be divided into two portions, one of which may be submitted for analysis and the other preserved by freezing for confirmatory analysis or reanalysis [30].

Stability: Resistance to decomposition or other chemical changes, or to physical disintegration [36].

Standard addition: Addition of a known amount of a pure component supposed to be present as a constituent of the specimen or sample in order to verify and quantitate this component [29]. Operationally, a measurement is made on the specimen or sample, a known amount of the desired constituent is added, the modified specimen is remeasured, and the amount of the constituent originally present is determined by proportionation [56].

Standard analyte: Well-defined substance in its highest available purity to be used as a reference in the analysis.

Standard consensus method: See Reference method. 
Standard deviation: Statistic that shows the spread or dispersion of scores in a distribution of scores. It is calculated by taking the square root of the variance [20]. It is applicable to all kinds of repeated measurements, e.g. between-batch, within-batch, repeatability and reproducibility.

Standard operating procedure (SOP): Written procedure which describes how to perform certain laboratory activities [32].

Standard solution: Solution of known concentration prepared from characterized material.

Statistical control: A procedure is in statistical control when results consistently fall within established control limits, that is, with constant mean and variance [16]. Statistical control should be monitored graphically with control charts [14].

Statistical correlation: Extent to which two or more things are related to one another. This is usually expressed as a correlation coefficient [20].

Statistical significance: Said of a value or measure of a variable when it is larger or smaller than would be expected by chance alone. Statistical significance does not necessarily imply practical significance [20].

Stock solution: Concentrated standard solution used to prepare calibrators.

Study: Experiment or set of experiments in order to obtain information on a particular subject.

Surrogate analyte: See Analyte.

Surveillance: Monitoring of certain activities to ensure that specified requirements have been fulfilled [4].

Survey: Study conducted among organizations to compile information on their activities or performance.

SWGDRUG: Scientific Working Group for the Analysis of Seized Drugs [57].

System suitability test: Validation of an analytical system (system suitability testing) tests a system against documented performance specifications, for a specific analytical method [58].

Systematic error: See Error.

Target analyte: See Analyte.

Target value: See Value. 
t-distribution: See Distribution.

Technical manager: Person with responsibility for specified technical operations, the provision of the resources needed to ensure the required quality of these operations, the investigation and resolution of technical problems, and the evaluation of instrument calibration and maintenance records

Technical procedure: Documented operation (procedure) carried out in a laboratory by trained technical or scientific personnel.

Technical review: Review of bench notes, data and other documents which form the basis for scientific conclusion. See also Peer review.

Technique: Scientific principle, for example gas chromatography or ultraviolet spectrometry, that can be used to provide data on the composition of a material. It is unusual to apply a technique directly to a test sample, as extraction and other steps are frequently required. A technique is therefore used in the last step of an analytical method which is usually the end-determination or end-step determination.

Test: Technical operation to determine one or more characteristics of or to evaluate the performance of a given product, material, equipment, organism, physical phenomenon, process or service according to a specified procedure [19].

Test linearity: Ability within a given range to obtain test results directly proportional to the concentration (amount) of analyte in the specimen or sample [59].

Test procedure: See Procedure.

Theoretical probability distribution: See Distribution.

Threshold: Particular, significant amount, level, or limit, at which something begins to happen or take effect. See Cut-off concentration.

Total error: See Error.

Traceable: See Traceability.

Traceable standard: Reference standard which also has the property of traceability. It will usually have a certificate of analysis giving details of the national or international standards used to determine its composition.

Traceability: Ability to trace the history, application or location of an entity by means of recorded identification [4]. See also Chain of custody. Alternatively, traceability is a property of the result of a measurement or the value of a standard whereby it can be related with a stated uncertainty, to stated references, usually national or international standards, i.e. through an unbroken chain of comparisons. In this context, The standards referred to here are measurement standards rather than written standards. 
Training record: Record maintained for each member of staff of their education, qualifications and training together with a list of tasks they are competent to perform and authorized to carry out, for example, to perform particular types of test, to issue test reports, to give opinions and interpretations and to operate particular types of equipment.

Trueness: Closeness of agreement between the average value obtained from a large series of test results and an accepted reference or true value. See also Accuracy and Bias.

True value: See Value.

t-test: See Significance test.

Type I and Type II errors: See Error.

Uncertainty: Parameter associated with the result of a measurement that characterizes the dispersion of the values that could reasonably be attributed to the analyte. The parameter may be, for example, a standard deviation (or a given multiple of it), or the width of a confidence interval. Alternatively, uncertainty is an estimate attached to a test result which characterizes the range of values within which the true value is asserted to lie.

Universe: See Population.

Upper limit of quantification (ULOQ): Highest amount of an analyte in a sample that can be quantitatively determined with an acceptable level of precision and accuracy.

Validated method: Method whose performance characteristics meet the specifications required by the intended use of the analytical results [40]. Some of the performance characteristics to be evaluated are limit of detection, limit of quantitation, linearity, precision, range, ruggedness, selectivity and specificity, and trueness.

Validation: Confirmation by examination and provision of objective evidence that the particular requirements for a specific intended use are fulfilled [4]. See also Method validation.

Equipment validation: see Performance verification.

Validation parameters: Key aspects of an analytical method which are evaluated for the purposes of method development and validation, including accuracy (bias), linearity, limit of detection, limit of quantitation, range, recovery, repeatability, reproducibility, ruggedness, and specificity (selectivity) [46]. See also Performance characteristics.

Value: Expression of a quantity in terms of a number and an appropriate unit of measurement [28]. 
Accepted reference value: Value that serves as an agreed-upon reference for comparison and which is derived as $(a)$ a theoretical or established value, based on scientific principles, $(b)$ an assigned or certified value, based on experimental work of some national or international organization, $(c)$ a consensus or certified value, based on collaborative experimental work under the auspices of a scientific or engineering group or $(d)$ when $(a),(b)$ and $(c)$ are not available, the experimentation of the (measurable) quantity, i.e. the mean of a specified population of measurements [11].

Assigned value: Best available estimate of the true value [60].

Consensus value: Value produced by a group of experts or referee laboratories using the best possible methods. It is an estimate of the true value.

Estimate value (statistical): Value(s) of population characteristic(s) obtained from sample data.

Target value: Numerical value of a measurement result, which has been designated as a goal for measurement quality [61].

True value: Value that characterizes a quantity perfectly defined in the conditions which exist when that quantity is considered. The true value of a quantity is an ideal concept and, in general, cannot be known exactly [28].

Variability: Spread or dispersion of scores in a group of scores; the tendency of each score to be unlike the others [20].

Variable: Generally, any quantity that varies. More precisely, a quantity which may take any one of a specified set of values [23].

Variance: Statistic that shows the spread or dispersion of scores in a distribution of scores. It is calculated by the sum of the squares of the differences between the individual values of a set and the arithmetic mean of the set, divided by one less than the number of values $[16,20]$.

Variate: In contradistinction to a variable, a variate is a quantity which may take any of the values of a specified set with a specified relative frequency or probability. It is often known as a random variable [23].

Verification: Confirmation by examination and provision of objective evidence that specified requirements have been fulfilled [4].

Warning limit: See Limit.

Working range: See Range

Working standard solutions: Standard solutions prepared by dilution of the stock solution containing the concentrations used to establish the calibration curve. 
z-distribution: See Distribution.

$z$-Score: Number of standard derivation units that separate a value from its mean [62]. 



\section{Bibliography/Reference list of source documents}

1. Guidance on the Implementation of a Quality Management System in Drug Testing Laboratories, ST/NAR/37, UNODC, 2009.

2. Guidance for the Validation of Analytical Methodology and Calibration of Equipment used for Testing of Illicit Drugs in Seized Materials and Biological Specimens, ST/NAR/41, UNODC, 2009

3. Glossary of Terms for Quality Assurance and Good Laboratory Practices, ST/ NAR/26, United Nations, 1995.

4. ISO/DIS 8402, (1991), Quality Management and Quality Assurance Vocabulary.

5. International Organization for Standardization, International Vocabulary of Basic and General Terms Used in Metrology (Geneva, 1984).

6. Scientific Working Group for the Analysis of seized drugs (SWGDRUG) Recommendations, 4 [th] Edition, 2008, available online at www.swgdrug.org/approved. htm.

7. EURACHEM Guide, The Fitness for Purpose of Analytical Methods: A laboratory Guide to Method Validation and Related Topics, 1998, available online at www.eurachem.org/guides/valid.pdf

8. UNDCP, "Report of the Consultative Meeting on Quality Assurance and Good Laboratory Practices”, Glasgow, 2-6 November 1992.

9. L. Huber, Validation of Computerized Analytical Systems, Interpharm Press Inc., Buffalo Grove, IL, 1996.

10. National Institute for Drug Abuse, Urine Testing for Drugs of Abuse, Research Monograph 73 (Rockville, Maryland, Department of Health and Human Services, 1986).

11. IUPAC, Compendium of Analytical Nomenclature, The Orange Book-3rd Edition, J. Inczedy, T. Lengyel, and A.M. Ure, Blackwell Science, 1998 [ISBN 0-632-05127-2], available online at http://old.iupac.org/publications/analytical_ compendium/

12. J. C. Miller and J. N. Miller, Statistics for Analytical Chemistry (Chichester and New York, Ellis Horwood, 1984). 
13. R. Wennig, Practical Compendium for Health Professionals: Drugs of Abuse Currently Used in Europe, Publication CEC/V/E/I/Lux 92 (Luxembourg, Commission of the European Communities, Health and Safety Directorate, 1992).

14. International Olympic Committee/Reference Materials Committee of ISO, "Quality control of analytical data produced in chemical laboratories", Publication 271, draft protocol presented to the Fifth International Symposium on the Harmonization of Internal Quality Assurance Schemes for Analytical Laboratories, Washington, D.C., 23 July 1993.

15. PONS English Language Dictionary (London, Collins Cobuild., 1987).

16. F. M. Garfield, Quality Assurance Principles for Analytical Laboratories (Arlington, Virginia, AOAC, 1991).

17. Clinical Pathology Accreditation (UK) Ltd, www.cpa-uk.co.uk/

18. American Society of Crime Laboratory Directors, Laboratory Accreditation Manual (Norfolk, Virginia, 1992).

19. ISO/International Electrotechnical Commission (IEC), Guide 25: General Requirements for the Competence of Calibration and Testing Laboratories (Geneva, 1990).

20. W. P. Voight, Dictionary of Statistics and Methodology: A Non-technical Guide for the Social Sciences (Thousand Oaks, California, Sage Publications, 1993).

21. European Community, Guideline Criteria for Reference Methods, BNL SP/Lab/ div (92) 5 (1992), p. 27.

22. ISO/International Electrotechnical Commission, Guide 2: General Terms and Their Definitions concerning Standardization and Related Activities (Geneva, 1991).

23. M. G. Kendall and W. R Buckland, A Dictionary of Statistical Terms (London, Longman Group, 1976).

24. C. Visher, A Comparison of Urinalysis Technologies for Drug Testing in Criminal Justice (Washington, D.C., National Institute of Justice and Bureau of Justice Assistance, 1991).

25. Society of Forensic Toxicologists Inc./American Academy of Forensic Sciences, Forensic Toxicology Laboratory Guidelines, 1991.

26. Chambers English Dictionary, W \& R Chambers Ltd., Edinburgh (1990).

27. K. Eckschlager, Errors, Measurement and Results in Chemical Analysis (London, Van Nostrand Reinhold, 1969).

28. International Union of Pure and Applied Chemistry, Spectrochemica Acta, 1978.

29. National Measurement Accreditation Service, NIS 46, Accreditation for Forensic Analysis and Examination (Teddington, Middlesex, 1992). 
30. B. S. Finkle, R. V. Blanke and J. M. Walsh, eds., Technical, Scientific and Procedural Issues of Employee Drug Testing (Rockville, Maryland, NIDA, 1990).

31. J. A. Timbrell, Introduction to Toxicology (London, Taylor and Francis, 1989).

32 OECD, The OECD Principles of Good Laboratory Practice, Environmental Monograph No. 45, Paris, 1992.

33. D. McCormick and A. Roach, Measurement, Statistics and Computation (Chichester, Wiley, 1988).

34. International Organization for Standardization, internet site at www.iso.org/iso/ home.htm

35. D.W. Tholen, ISO/IEC 17043: the new International Standard for proficiency testing, Accred Qual Assur 13:727-730 (2008).

36. Webster's Third New International Dictionary of the English Language (Springfield, Massachusetts, Merriam, 1971).

37. FAO/WHO, Codex Alimentarius Commission, Criteria to Limit the Number of False Positive and False Negative Results for Analytes Near the Limit of Determination, CX/MAS 92/15, Budapest, 1992.

38. NATA Technical Note 17-April 2009, Guidelines for the validation and verification of chemical test methods, accessed at www.nata.asn.au

39. IUPAC, Compendium of Chemical Technology, 2 [nd] Edition (the "Gold Book"), accessed online at http://goldbook.iupac.org/index.html

40. EURACHEM/WELAC Chemistry Working Group, Accreditation for Chemical Laboratories: Guidance on the Interpretation of the EN45000 Series of Standards and ISO/IEC Guide 25 (Teddington, Middlesex, EURACHEM/WELAC, 1993).

41. International Organization for Standardization/Development Information System 8402, Quality Management and Quality Assurance Vocabulary (Geneva, 1991).

42. D.R. Jenke, Chromatographic Method Validation: a review of current practices and procedures, I General concepts and guidelines. J. Liq. Chrom. \& Rel. Technol. 19, 719-736 (1996).

43. R. Caulcutt and R. Boddy, Statistics for Analytical Chemists (New York, Chapman and Hall, 1983).

44. W. Horwitz, "Protocol for the design, conduct and interpretation of collaborative studies", Pure and Applied Chemistry, vol. 60 (1988), pp. 855-864

45. P. C. Kelly, "Outlier detection in collaborative studies", Journal of the Association of Official Analytical Chemists, vol. 73 (1990), pp. 58-64

46. E. Prichard (Ed.) Trace Analysis, pp 32-39 (Cambridge, Royal Society of Chemistry, 1996). 
47. B. G. Katzungt and A. J. Trevor, Pharmacology (London, Prentice Hall International, 1993).

48. G.T. Wernimont in W. Spendley (Ed.), Use of Statistics to Develop and Evaluate Methods, Association of Official Analytical Chemists, Arlington, VA, p 78-82 (1985).

49. F.H.C. Marriot and M. G. Kendall, A Dictionary of Statistical Terms, 5th ed. (Harlow, Essex and New York, Longman Scientific \& Technical/Wiley, 1990).

50. J. M. F. Nogueira, C. A. Nieto de Castro, Leopoldo Cortez, EPTIS: The new European database of proficiency testing schemes for analytical laboratories. Trends in Analytical Chemistry, 20, 457-461,(2001).

51. J. K. Taylor, Quality Assurance of Chemical Measurements (Boca Raton, Florida, Lewis, 1987).

52. L. Stebbing, Quality Assurance: the Route to Efficiency and Competitiveness, 3rd ed. (Chichester and New York, Ellis Horwood, 1993).

53. A. C. Moffatt and M. D. Osselton, Characterization of quantitative methods for the analysis of drugs in blood in forensic toxicology, Bulletin of the International Association of Forensic Toxicologists, vol. 20, No. 3 (1990), pp. 36-41.

54. World Health Organization, Good Laboratory Practices in Governmental Drug Control Laboratories, WHO/PHARM/84.512/Rev.2, 1984.

55. R.S. Galen and S.R. Gambino, Beyond Normality: The Predictive Value and Efficiency of Medical Diagnoses, John Wiley \& Sons, New York, 1975.

56. I. M. Kolthoff, P. J. Elving and E. J. Meehan, eds., Treatise on Analytical Chemistry, 2nd ed. (New York, John Wiley, 1978).

57. SWGDRG, www.swgdrug.org

58. L. Huber, Validation of Computerized Analytical Systems, Interpharm Press Inc., Buffalo Grove, IL, 1996.

59. Commission of the European Communities, Directorate III, Addendum, Guidelines on the Quality, Safety and Efficacy of Medical Products for Human Use, Vol. III, Publication CEC/III/EN/9104/90 (Brussels, 1990).

60. Analytical Methods Committee, "Proficiency testing of analytical laboratories: organization and statistical assessment", Analyst, vol. 117 (1992), pp. 97-117.

61. ISO/REMCO, "The international harmonized protocol for the proficiency testing of (chemical) analytical laboratories”, Publication 263, Geneva, 1992.

62. W. Horowitz and R. Albert, "Experience with the IUPAC-1987 Harmonized Protocol for Method-Performance Studies: suggestions for revision and application to internal quality control systems", Quality Assurance for Analytical Laboratories, M. Parkany, ed. (Cambridge, Royal Society of Chemistry, 1993). 


$$
\begin{aligned}
& \text { كيفية الحصول على منشورات الأمم المتحدة }
\end{aligned}
$$

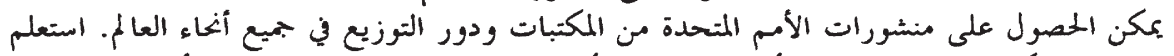

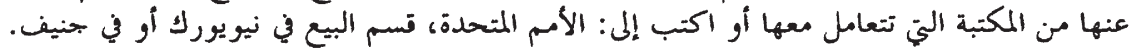

$$
\begin{aligned}
& \text { 如何购取联合国出版物 }
\end{aligned}
$$

联合国出版物在全世界各地的书店和经管处均有发售。请向书店诲问或写信到纽约或日内瓦的联合国销售组。

\section{HOW TO OBTAIN UNITED NATIONS PUBLICATIONS}

United Nations publications may be obtained from bookstores and distributors throughout the world. Consult your bookstore or write to: United Nations, Sales Section, New York or Geneva.

\section{COMMENT SE PROCURER LES PUBLICATIONS DES NATIONS UNIES}

Les publications des Nations Unies sont en vente dans les librairies et les agences dépositaires du monde entier. Informez-vous auprès de votre libraire ou adressez-vous à: Nations Unies, Section des ventes, New York ou Genève.

\section{КАК ПОЛУЧИТЬ ИЗДАНИЯ ОРГАНИЗАЦИИ ОБЬЕДИНЕННЫХ НАЦИЙ}

Издания Организации Объединенных Наций можно купить в книжных магазинах и агентствах во всех районах мира. Наводите справки об изданиях в вашем книжном магазине или пишите по адресу: Организация Объединенных Наций, Секция по продаже изданий, Нью-Йорк или Женева.

\section{CÓMO CONSEGUIR PUBLICACIONES DE LAS NACIONES UNIDAS}

Las publicaciones de las Naciones Unidas están en venta en librerías y casas distribuidoras en todas partes del mundo. Consulte a su librero o diríjase a: Naciones Unidas, Sección de Ventas, Nueva York o Ginebra. 
Vienna International Centre, PO Box 500, 1400 Vienna, Austria Tel.: (+43-1) 26060-0, Fax: (+43-1) 26060-5866, www.unodc.org

United Nations publication

Printed in Austria

Sales No. E.10.XI.7

ST/NAR/26/Rev.1

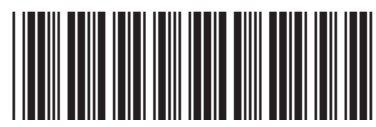

V.09-87608-December 2009-440
USD 15

ISBN 978-92-1-148253-9

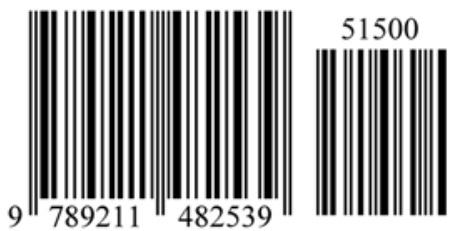

